\title{
THE FINITE SUM OF THE PRODUCTS OF TWO TOEPLITZ OPERATORS
}

\author{
XUANHAO DING
}

\author{
(Received 27 November 2006; accepted 29 August 2007)
}

Communicated by J. J. Koliha

\begin{abstract}
We consider in this paper the question of when the finite sum of products of two Toeplitz operators is a finite-rank perturbation of a single Toeplitz operator on the Hardy space over the unit disk. A necessary condition is found. As a consequence we obtain a necessary and sufficient condition for the product of three Toeplitz operators to be a finite-rank perturbation of a single Toeplitz operator.
\end{abstract}

2000 Mathematics subject classification: primary 47B35.

Keywords and phrases: Hardy space, Toeplitz operators, finite-rank operators.

\section{Introduction}

Let $D$ be the open unit disk in the complex plane and $\partial D$ the unit circle. Let $d \sigma(z)$ be the normalized Lebesgue measure on the unit circle $\partial D$. Let $L^{q}$ denote the Lebesgue $q$-square integrable functions on the unit circle and let $L^{\infty}$ be the space of essentially bounded functions on the unit circle. The Hardy space $H^{2}$ is the Hilbert space consisting of the analytic functions on the unit disk $D$ that are also in $L^{2} . H^{\infty}$ denotes the set of bounded analytic functions on the unit disk. Let $P$ be the orthogonal projection from $L^{2}$ onto $H^{2}$. For $f \in L^{\infty}$, the Toeplitz operator $T_{f}$ and the Hankel operator $H_{f}$ with symbol $f$ are defined by $T_{f} h=P(f h)$ and $H_{f} h=(1-P)(f h)$ for $h$ in $H^{2}$. A bounded linear operator on the Hilbert space $H$ is said to have finite rank if the closure of the range of the operator has finite dimension. As is well known, Hankel and Toeplitz operators are closely related by the following important fact:

$$
T_{f g}-T_{f} T_{g}=H_{\bar{f}}^{*} H_{g} .
$$

Studying the Toeplitz algebra has shed light on the theory of Toeplitz operators $[3,4,8]$. We know that the algebra of finite sums of finite products of Toeplitz

This work was partially supported by the National Natural Science Foundation of China (10361003) and Guangxi Natural Science Foundation (0542046).

(C) 2009 Australian Mathematical Society 1446-7887/2009 \$16.00 
operators is dense in the Toeplitz algebra. Guo and Zheng [7] and Gu [5] have shown that a finite sum of finite products of Toeplitz operators can be written as a finite sum of products of two Toeplitz operators. Conditions that characterize when a finite sum of finite products of a Toeplitz operator is a compact perturbation of a Toeplitz operator were found by Guo and Zheng [7].

Another motivation is the result of $\mathrm{Gu}$ [6], which states that if an operator $X$ on $H^{2}$ is such that $X-T_{\theta}^{*} X T_{\theta}$ is of finite rank for every inner function $\theta$, then $X=T_{\psi}+F$ where $\psi \in L^{\infty}$ and $F$ is a finite-rank operator on $H^{2}$. In particular, if we set $X=\sum_{i=1}^{n} H_{\overline{z f_{i}(z)}}^{*} H_{\overline{z g_{i}(z)}}$, then $X-T_{z}^{*} X T_{z}=\sum_{i=1}^{n} f_{i} \otimes g_{i}$ is of finite rank. However, we do not know under what conditions the finite sum of two Hankel operator is of finite rank for general symbols. It is easy to see that

$$
\sum_{i=1}^{n} T_{f_{i}} T_{g_{i}}-T_{\sum_{i=1}^{n} f_{i} g_{i}}=\sum_{i=1}^{n} H_{f_{i}}^{*} H_{g_{i}} .
$$

A natural question arises: When is the finite sum of products of two Toeplitz operators a finite-rank perturbation of a single Toeplitz operator?

In Section 2, we will give a necessary condition for the finite sum of products of two Toeplitz operators to be a finite-rank perturbation of a Toeplitz operator.

In Section 3, we will give some interesting consequences.

\section{Necessary condition}

We need to introduce some notation. For $x, y \in H^{2}, x \otimes y$ is the operator of rank one defined by

$$
x \otimes y(f)=\langle f, y\rangle x
$$

for every $f \in H^{2}$. It is easy to see that $(x \otimes y)^{*}=y \otimes x$.

Let $A$ be a finite-rank operator on $H^{2}$, where $A$ has rank $k$. Then there are vectors $x_{j}, y_{j}$ in $H^{2}$ with $\operatorname{dim}\left\{x_{j}\right\}=\operatorname{dim}\left\{y_{j}\right\}=k$ such that $A=\sum_{j=1}^{k} x_{j} \otimes y_{j}$.

Although our main concern is with bounded Toeplitz operators and Hankel operators, since a product of $m(\geq 3)$ Toeplitz operators can be decomposed into the product of two Toeplitz operators with perhaps unbounded symbols, we will need to make use of densely defined unbounded Toeplitz operators and Hankel operators. Given two operators $S_{1}$ and $S_{2}$ densely defined on $H^{2}$, we say that $S_{1}=S_{2}$ if $S_{1} P=S_{2} P$ for each analytic polynomial $P$.

Note that $\bigcap_{1<q<\infty} L^{q}$ is an algebra, that is, both $f g$ and $f+g$ are in $\bigcap_{1<q<\infty} L^{q}$ if $f$ and $g$ are in $\bigcap_{1<q<\infty} L^{q}$. In addition, the Hardy projections $P$ and $1-P$ are bounded on $L^{q}$ for $1<q<\infty$. Naturally, we consider the symbols of Toeplitz operators in $\bigcap_{1<q<\infty} L^{q}$. For $f \in \bigcap_{1<q<\infty} L^{q}$, let $f^{+}=P f$, the analytic part of $f$, and let $f^{-}=(1-P) f$, the conjugate analytic part of $f$. It is well known that $T_{\bar{z}} T_{f} T_{z}=T_{f}$. Our main result is the following theorem. 
Theorem 2.1. For $f_{i}, g_{i}, h$ in $\bigcap_{1<q<\infty} L^{q}(i=1,2, \ldots, n)$, if $\sum_{i=1}^{n} T_{f_{i}} T_{g_{i}}-T_{h}$ is a finite-rank operator, then there are analytic polynomials $A_{i}(z), B_{i}(z)$ with $\max \left\{\operatorname{deg} A_{i}(z)\right\}=k$ and $\max \left\{\operatorname{deg} B_{i}(z)\right\}=k$, not all of which are zero, such that

$$
\sum_{i=1}^{n} A_{i} \bar{f}_{i} \in H^{2}
$$

or

$$
\sum_{i=1}^{n} B_{i} g_{i} \in H^{2}
$$

PROOF. Let $K$ be the rank of $\sum_{i=1}^{n} T_{f_{i}} T_{g_{i}}-T_{h}$. We prove the result by induction on the rank $K$.

Assume that the rank $K=0$. Then

$$
\sum_{i=1}^{n} T_{f_{i}} T_{g_{i}}=T_{h}
$$

If one of the $\bar{f}_{i}$ or one of the $g_{i}$ is in $H^{2}$, then obviously there are constants $A_{i}, B_{i}$ with $\sum_{i=1}^{n}\left|A_{i}\right|>0$ and $\sum_{i=1}^{n}\left|B_{i}\right|>0$ such that

$$
\sum_{i=1}^{n} A_{i} \bar{f}_{i} \in H^{2}
$$

or

$$
\sum_{i=1}^{n} B_{i} g_{i} \in H^{2}
$$

If none of the $\bar{f}_{i} \in H^{2}$ and none of the $g_{i} \in H^{2}$, let $K_{\lambda}(z)=(1 /(1-\bar{\lambda} z))$ be the reproducing kernel at $\lambda \in D$. Noting that $1-T_{z} T_{\bar{z}}=1 \otimes 1$, it follows that

$$
\begin{aligned}
\left(T_{\bar{z} f_{1}} 1 \otimes T_{\bar{z} \bar{g}_{1}} 1+\cdots+T_{\bar{z} f_{n}} 1 \otimes T_{\bar{z} \bar{g}_{n}} 1\right) & =T_{\bar{z}} \sum_{i=1}^{n} T_{f_{i}}\left(1-T_{z} T_{\bar{z}}\right) T_{g_{i}} T_{z} \\
& =T_{\bar{z}} \sum_{i=1}^{n} T_{f_{i}} T_{g_{i}} T_{z}-\sum_{i=1}^{n} T_{\bar{z}} T_{f_{i}} T_{z} T_{\bar{z}} T_{g_{i}} T_{z} \\
& =T_{\bar{z}} \sum_{i=1}^{n} T_{f_{i}} T_{g_{i}} T_{z}-\sum_{i=1}^{n} T_{f_{i}} T_{g_{i}} \\
& =T_{\bar{z}} T_{h} T_{z}-T_{h}=0 .
\end{aligned}
$$

Then

$$
\sum_{i=1}^{n} \overline{T_{\bar{z}} \bar{g}_{i} 1(\lambda)} T_{\bar{z} f_{i}} 1=\sum_{i=1}^{n} T_{\bar{z} f_{i}} 1 \otimes T_{\bar{z} \bar{g}_{i}} 1\left(K_{\lambda}\right)=0
$$


It is easy to see that $T_{\bar{z} \bar{g}_{i}} 1=0$ if and only if $g_{i} \in H^{2}$ and $T_{\bar{z}} f_{i} 1=0$ if and only if $\bar{f}_{i} \in H^{2}$. Hence there is a $\lambda_{0} \in D$ such that $A_{i}=T_{\bar{z} \bar{g}_{i}} 1\left(\lambda_{0}\right) \neq 0$ for all $1 \leq i \leq n$. Thus

$$
\sum_{i=1}^{n} \bar{A}_{i} T_{\bar{z} f_{i}} 1=0
$$

implies that

$$
\sum_{i=1}^{n} A_{i} \bar{f}_{i} \in H^{2}
$$

Next we assume that the result is true if the rank $k$ is less than $K$. We need to show that the result is true for $k=K$.

We write

$$
\sum_{i=1}^{n} T_{f_{i}} T_{g_{i}}-T_{h}=\sum_{j=1}^{k} x_{j} \otimes y_{j},
$$

where $x_{j}, y_{j}$ are in $H^{2}$ and $\operatorname{dim}\left\{x_{j}\right\}=\operatorname{dim}\left\{y_{j}\right\}=K$.

We have

$$
\begin{aligned}
\sum_{i=1}^{n} T_{\bar{z} f_{i}} 1 \otimes T_{\bar{z} \bar{g}_{i}} 1 & =T_{\bar{z}}\left\{\sum_{i=1}^{n} T_{f_{i}}\left(1-T_{z} T_{\bar{z}}\right) T_{g_{i}}\right\} T_{z} \\
& =T_{\bar{z}} \sum_{i=1}^{n} T_{f_{i}} T_{g_{i}} T_{z}-\sum_{i=1}^{n} T_{f_{i}} T_{g_{i}} \\
& =T_{\bar{z}}\left(T_{h}+\sum_{j=1}^{k} x_{j} \otimes y_{j}\right) T_{z}-\left(T_{h}+\sum_{j=1}^{k} x_{j} \otimes y_{j}\right) \\
& =\sum_{j=1}^{k} T_{\bar{z}} x_{j} \otimes T_{\bar{z}} y_{j}-\sum_{j=1}^{k} x_{j} \otimes y_{j} .
\end{aligned}
$$

That is,

$$
\sum_{i=1}^{n} T_{\bar{z} f_{i}} 1 \otimes T_{\overline{z g}} 1=\sum_{j=1}^{k} T_{\bar{z}} x_{j} \otimes T_{\bar{z}} y_{j}-\sum_{j=1}^{k} x_{j} \otimes y_{j} .
$$

If $T_{\bar{z}} y_{1}, \ldots, T_{\bar{z}} y_{k}$ are linearly dependent, without loss of generality, we may assume that

$$
T_{\bar{z}} y_{k}=c_{1} T_{\bar{z}} y_{1}+\cdots+c_{k-1} T_{\bar{z}} y_{k-1},
$$

for some constants $c_{1}, \ldots, c_{k-1}$. Then

$$
\sum_{i=1}^{n} T_{\bar{z} f_{i}} T_{z g_{i}}=T_{h}+\sum_{j=1}^{k-1} T_{\bar{z}}\left(x_{j}+\overline{c_{j}} x_{k}\right) \otimes T_{\bar{z}} y_{j} .
$$


Thus the rank of $\sum_{i=1}^{n} T_{\bar{z}} f_{i} T_{z g_{i}}-T_{h}$ is at most $K-1$. So, by the induction hypothesis, there exist analytic polynomials $a_{i}(z)$ and $b_{i}(z)$ with $\max \left\{\operatorname{deg} a_{i}(z)\right\} \leq$ $K-1, \max \left\{\operatorname{deg} b_{i}(z)\right\} \leq K-1$, and $\sum_{i=1}^{n}\left|a_{i}\right| \sum_{i=1}^{n}\left|b_{i}\right|>0$ such that

$$
\sum_{i=1}^{n} a_{i}(z) z \bar{f}_{i} \in H^{2}
$$

or

$$
\sum_{i=1}^{n} b_{i}(z) z g_{i} \in H^{2}
$$

Let $l=\max \left\{\operatorname{deg} a_{i}(z)\right\}$ or $l=\max \left\{\operatorname{deg} b_{i}(z)\right\} . \quad$ Then $A_{i}=z^{k-l} a_{i}(z)$ and $B_{i}=$ $z^{k-l} b_{i}(z)$ are both analytic polynomials with $\max \left\{\operatorname{deg} A_{i}\right\}=\max \left\{\operatorname{deg} B_{i}\right\}=k$, $\sum_{i=1}^{n}\left|A_{i}\right| \sum_{i=1}^{n}\left|B_{i}\right| \neq 0$ such that

$$
\sum_{i=1}^{n} A_{i} \bar{f}_{i} \in H^{2}
$$

or

$$
\sum_{i=1}^{n} B_{i} g_{i} \in H^{2}
$$

Thus the result is true in this case.

If $T_{\bar{z}} x_{1}, \ldots, T_{\bar{z}} x_{k}$ are linearly dependent, by the same argument as above, we obtain that the result is true.

To finish the proof, we may assume that $T_{\bar{z}} y_{1}, \ldots, T_{\bar{z}} y_{k}$ are linearly independent and $T_{\bar{z}} x_{1}, \ldots, T_{\bar{z}} x_{k}$ are linearly independent.

Applying $T_{\bar{z}} y_{l}$ to both sides of (2.1) gives that

$$
\sum_{i=1}^{n}\left\langle T_{\bar{z}} y_{l}, T_{\bar{z} g_{i}} 1\right\rangle T_{\bar{z} f_{i}} 1=\sum_{j=1}^{k}\left\langle T_{\bar{z}} y_{l}, T_{\bar{z}} y_{j}\right\rangle T_{\bar{z}} x_{j}-\sum_{j=1}^{k}\left\langle T_{\bar{z}} y_{l}, y_{j}\right\rangle x_{j},
$$

for $l=1,2, \ldots, k$.

Let $a_{l j}=\left\langle T_{\bar{z}} y_{l}, T_{\bar{z}} y_{j}\right\rangle, b_{l j}=\bar{z} a_{l j}-\left\langle T_{\bar{z}} y_{l}, y_{j}\right\rangle, c_{l j}=\left\langle T_{\bar{z}} y_{l}, T_{\bar{z} \bar{g}_{i}} 1\right\rangle$. Since $T_{\bar{z}} x_{j}=$ $\bar{z} x_{j}-\bar{z} x_{j}(0)$,

$$
\begin{aligned}
\left(\begin{array}{cccc}
c_{11} & c_{12} & \cdots & c_{1 n} \\
c_{21} & c_{22} & \cdots & c_{2 n} \\
\cdots & \cdots & \cdots & \cdots \\
c_{k 1} & c_{k 2} & \cdots & c_{k n}
\end{array}\right)\left(\begin{array}{c}
T_{\bar{z} f_{1}} 1 \\
T_{\bar{z} f_{2}} 1 \\
\vdots \\
T_{\bar{z} f_{n} 1}
\end{array}\right) \\
\quad=\left(\begin{array}{cccc}
b_{11} & b_{12} & \cdots & b_{1 k} \\
b_{21} & b_{22} & \cdots & b_{2 k} \\
\cdots & \cdots & \cdots & \cdots \\
b_{k 1} & b_{k 2} & \cdots & b_{k k}
\end{array}\right)\left(\begin{array}{c}
x_{1} \\
x_{2} \\
\vdots \\
x_{k}
\end{array}\right)-\left(\begin{array}{cccc}
a_{11} & a_{12} & \cdots & a_{1 k} \\
a_{21} & a_{22} & \cdots & a_{2 k} \\
\cdots & \cdots & \cdots & \cdots \\
a_{k 1} & a_{k 2} & \cdots & a_{k k}
\end{array}\right)\left(\begin{array}{c}
x_{1}(0) \\
x_{2}(0) \\
\vdots \\
x_{k}(0)
\end{array}\right) \bar{z}
\end{aligned}
$$


That is,

$$
C T_{\bar{z} f} 1=B X-\bar{z} A X(0)
$$

where $C=\left(c_{l j}\right), B=\left(b_{l j}\right), A=\left(a_{l j}\right), T_{\bar{z} f} 1=\left(T_{\bar{z} f_{1}} 1, \ldots, T_{\bar{z} f_{k}} 1\right)^{\mathrm{T}}, X=\left(x_{1}, \ldots, x_{k}\right)^{\mathrm{T}}$, $X(0)=\left(x_{1}(0), \ldots, x_{k}(0)\right)^{\mathrm{T}}$. The determinant of the matrix $B=\left(b_{l j}\right)_{k \times k}$ is

$$
D(z)=\operatorname{det}\left(b_{l j}\right)=a \bar{z}^{k}+a_{1} \bar{z}^{k-1}+\cdots+a_{k},
$$

where $a=\operatorname{det}\left(a_{l j}\right)$ is the Gram determinant of vectors $T_{\bar{z}} y_{1}, T_{\bar{z}} y_{2}, \ldots, T_{\bar{z}} y_{k}$. Since $T_{\bar{z}} y_{1}, T_{\bar{z}} y_{2}, \ldots, T_{\bar{z}} y_{k}$ are linearly independent, $a=\operatorname{det}\left(a_{l j}\right) \neq 0$, and $a_{i}$ are constants. Hence $\operatorname{deg} D(z)=k$, and $D(z)$ is a co-analytic polynomial in $z$.

The adjoint of the matrix $B$ is

$$
\operatorname{adj} B=\left(\begin{array}{cccc}
B_{11} & B_{21} & \cdots & B_{k 1} \\
B_{12} & B_{22} & \cdots & B_{k 2} \\
\cdots & \cdots & \cdots & \cdots \\
B_{1 k} & B_{2 k} & \cdots & B_{k k}
\end{array}\right),
$$

where $B_{l j}$ denotes the cofactor of $b_{l j}$ and it is a co-analytic polynomial in $z$ with degree at most $k-1$.

So

$$
(\operatorname{adj} B) C T_{\bar{z} f} 1=D(z) X-(\operatorname{adj} B) A X(0) \bar{z} .
$$

Let

$$
\left(C_{l i}(z)\right)=(\operatorname{adj} B) C,
$$

where $C_{l i}(z)$ are co-analytic polynomials in $z$ with degree at most $k-1$.

Applying the projection $P$ to both sides of the above equation gives that

$$
P\left[\left(C_{l i}(z)\right) T_{\bar{z} f} 1\right]=P D(z) X .
$$

That is,

$$
\left(\begin{array}{c}
T_{\bar{z} \sum_{i=1}^{n} c_{1 i}(z) f_{i}}{ }^{1} \\
\vdots \\
T_{\bar{z} \sum_{i=1}^{n} c_{k i}(z) f_{i}} 1
\end{array}\right)=\left(\begin{array}{c}
T_{D(z)} x_{1} \\
\vdots \\
T_{D(z)} x_{k}
\end{array}\right) .
$$

By the same argument, we also have

$$
\left(\begin{array}{c}
T_{\bar{z} \sum_{i=1}^{n} u_{1 i}(z) \overline{g_{i}}} 1 \\
\vdots \\
T_{\bar{z} \sum_{i=1}^{n} u_{k i}(z) \overline{g_{i}}} 1
\end{array}\right)=\left(\begin{array}{c}
T_{E(z)} y_{1} \\
\vdots \\
T_{E(z)} y_{k}
\end{array}\right),
$$

where $u_{l i}(z)$ are co-analytic polynomials in $z$ with degree at most $k-1$ and $E(z)$ is a co-analytic polynomial in $z$ with degree $k$. 
If $T_{\overline{z g_{1}}} 1, T_{\overline{z g_{2}}} 1, \ldots, T_{\overline{z g_{n}}} 1, y_{1}, y_{2}, \ldots, y_{k}$ are linearly dependent, then there exist constants $a_{i}, b_{i}$, not all zero, such that

$$
\sum_{i=1}^{n} a_{i} T_{\overline{z g_{i}}} 1+\sum_{j=1}^{k} b_{j} y_{j}=0 .
$$

One of the $a_{1}, a_{2}, \ldots, a_{n}$ must be nonzero since $y_{1}, \ldots, y_{k}$ are linearly independent. Without loss of generality, assume that

$$
T_{\overline{z g_{n}}} 1=a_{1} T_{\overline{z g_{1}}} 1+\cdots+a_{n-1} T_{\overline{z g_{n-1}}} 1+b_{1} y_{1}+\cdots b_{k} y_{k} .
$$

Then

$$
\begin{aligned}
T_{E(z)} T_{\overline{z g n}} 1 & =T_{E(z) \overline{z g_{n}}} 1 \\
& =\sum_{i=1}^{n-1} a_{i} T_{\bar{z} E(z) \overline{g_{i}}} 1+\sum_{j=1}^{k} b_{j} T_{E(z)} y_{j} \\
& =\sum_{i=1}^{n-1} a_{i} T_{\bar{z} E(z) \overline{g_{i}}} 1+\sum_{j=1}^{k} b_{j} T_{\sum_{i=1}^{n} u_{j i}(z) \overline{z g_{i}}} 1 \\
& =\sum_{i=1}^{n-1} a_{i} T_{\bar{z} E(z) \overline{g_{i}}} 1+\sum_{i=1}^{n} T_{\sum_{j=1}^{k} b_{j} u_{j i}(z) \overline{z g_{i}}} 1 .
\end{aligned}
$$

Therefore,

$$
T_{\bar{z}}\left\{\left[E(z)-\sum_{j=1}^{k} b_{j} u_{j n}(z)\right] \overline{g_{n}}-\sum_{i=1}^{n-1}\left[a_{i} E(z)+\sum_{j=1}^{k} b_{j} u_{j i}(z)\right] \overline{g_{i}}(z)\right\} 1=0 .
$$

From this equation, it follows that

$$
\overline{\left[E(z)-\sum_{j=1}^{k} b_{j} u_{j n}(z)\right]} g_{n}-\sum_{i=1}^{n-1} \overline{\left[a_{i} E(z)+\sum_{j=1}^{k} b_{j} u_{j i}(z)\right]} g_{i} \in H^{2} .
$$

Let

$$
\begin{gathered}
B_{n}(z)=\overline{\left[E(z)-\sum_{j=1}^{k} b_{j} u_{j n}(z)\right]}, \\
B_{i}(z)=-\overline{\left[a_{i} E(z)+\sum_{j=1}^{k} b_{j} u_{j i}(z)\right]}, \quad 1 \leq i \leq n-1 .
\end{gathered}
$$

Then $B_{l}(z)$ are analytic polynomials in $z$ with degree $B_{n}(z)=k, \operatorname{deg} B_{i}(z) \leq k$, $1 \leq i \leq n-1$, and $\sum_{i=1}^{n} B_{i} g_{i} \in H^{2}$. This is the result as desired. 
By the same argument, if $T_{\bar{z} f_{1}} 1, T_{\bar{z} f_{2}} 1, \ldots, T_{\bar{z} f_{n}} 1, x_{1}, \ldots, x_{n}$ are linearly dependent, we also have that there exist analytic polynomials $A_{i}(z)$ with $\max \left\{\operatorname{deg} A_{i}(z)\right\}=k$ such that

$$
\sum_{i=1}^{n} A_{i} \bar{f}_{i} \in H^{2}
$$

Next we assume that $T_{\bar{z} f_{1}} 1, \ldots, T_{\bar{z} f_{n}} 1, x_{1}, \ldots, x_{k}$ are linearly independent and $T_{\overline{z g} 1} 1, \ldots, T_{\overline{z g n}} 1, y_{1}, \ldots, y_{k}$ are also linearly independent. We will derive a contradiction.

First we claim that

$$
\operatorname{dim} \operatorname{span}\left\{x_{1}, \ldots, x_{k}, T_{\bar{z}} x_{1}, \ldots, T_{\bar{z}} x_{k}\right\} \geq k+n .
$$

In fact, since $T_{\overline{z g_{1}}} 1, \ldots, T_{\overline{z g_{n}}} 1$ are linearly independent, there is a vector $\xi \in H^{2}$ such that $\left\langle\xi, T_{\overline{z g_{i}}} 1\right\rangle=1$ and $\left\langle\xi, T_{\bar{z} g_{j}} 1\right\rangle=0$ for all $j \neq i$.

Hence

$$
T_{\bar{z}} f_{i} 1=\sum_{j=1}^{k}\left\langle\xi, T_{\bar{z}} y_{j}\right\rangle T_{\bar{z}} x_{j}-\sum_{j=1}^{k}\left\langle\xi, y_{j}\right\rangle x_{j},
$$

by (2.1). This implies that $T_{\bar{z} f_{i}} 1 \in \operatorname{span}\left\{x_{1}, \ldots, x_{k}, T_{\bar{z}} x_{1}, \ldots, T_{\bar{z}} x_{k}\right\}$.

This gives

$$
\operatorname{span}\left\{T_{\bar{z} f_{1}} 1, \ldots, T_{\bar{z} f_{n}} 1, x_{1}, \ldots, x_{k}\right\} \subseteq \operatorname{span}\left\{x_{1}, \ldots, x_{k}, T_{\bar{z}} x_{1}, \ldots, T_{\bar{z}} x_{k}\right\} .
$$

Thus

$$
\begin{aligned}
\operatorname{dim} \operatorname{span}\left\{x_{1}, \ldots, x_{k}, T_{\bar{z}} x_{1}, \ldots, T_{\bar{z}} x_{k}\right\} & \geq \operatorname{dim} \operatorname{span}\left\{T_{\bar{z} f_{1}} 1, \ldots, T_{\bar{z} f_{n}} 1, x_{1}, \ldots, x_{k}\right\} \\
& =k+n .
\end{aligned}
$$

Since

$$
\operatorname{dim} \operatorname{span}\left\{T_{\bar{z}} x_{1}, \ldots, T_{\bar{z}} x_{k}\right\}=k<k+n,
$$

there is a nonzero vector $\xi$ in $\operatorname{span}\left\{x_{1}, \ldots, x_{k}, T_{\bar{z}} x_{1}, \ldots, T_{\bar{z}} x_{k}\right\}$ such that

$$
\xi \perp\left\{T_{\bar{z}} x_{1}, \ldots, T_{\bar{z}} x_{k}\right\} .
$$

By (2.1),

$$
\sum_{i=1}^{n}\left\langle\xi, T_{\bar{z} f_{i}} 1\right\rangle T_{\overline{z g i}} 1=-\sum_{j=1}^{k}\left\langle\xi, x_{j}\right\rangle y_{j} .
$$

Not all of $\left\{\left\langle\xi, x_{j}\right\rangle\right\}_{j=1}^{k}$ are zero since

$$
\xi \in \operatorname{span}\left\{x_{1}, \ldots, x_{k}, T_{\bar{z}} x_{1}, \ldots, T_{\bar{z}} x_{k}\right\} .
$$


Otherwise $\xi$ is orthogonal to $\left\{x_{1}, \ldots, x_{k}, T_{\bar{z}} x_{1}, \ldots, T_{\bar{z}} x_{k}\right\}$, which would imply that $\xi=0$. This is a contradiction. Also

$$
\sum_{i=1}^{n}\left\langle\xi, T_{\bar{z} f_{i}} 1\right\rangle T_{\bar{z} \bar{g}_{i}} 1+\sum_{i=1}^{n}\left\langle\xi, x_{j}\right\rangle y_{j}=0
$$

where at least one coefficient $\left\langle\xi, x_{j}\right\rangle$ is different from zero. Thus, the vectors

$$
T_{\bar{z} g_{1}} 1, \ldots, T_{\bar{z} g_{n}} 1, y_{1}, \ldots, y_{k},
$$

are linearly dependent. We have obtained a contradiction to complete the proof.

Remark. Let $f_{i}$ and $g_{i}$ all be in $L^{\infty}$. Then the result of Theorem 2.1 is that there are no all-zero analytic polynomials $A_{i}(z), B_{i}(z)$ with $\max \left\{\operatorname{deg} A_{i}(z)\right\}=k$ and $\max \left\{\operatorname{deg} B_{i}(z)\right\}=k$ such that

$$
\sum_{i=1}^{n} A_{i} \bar{f}_{i} \in H^{\infty}
$$

or

$$
\sum_{i=1}^{n} B_{i} g_{i} \in H^{\infty}
$$

\section{Consequences}

In this section, we will obtain some necessary and sufficient conditions. For convenience, we write $A=B \bmod (F)$ to denote that the operator $A-B$ is a finiterank operator. One of the first results about Hankel matrices was Kronecker's theorem that describes the Hankel matrices of finite rank. Kronecker's theorem states that, for $f \in L^{\infty}, H_{f}$ is of finite rank if and only if $f$ is the sum of an analytic function $h$ and a rational function $r(z)$ whose poles are not on the unit circle. The following theorem is another form [9] of Kronecker's theorem, which we will use often in this section.

Theorem 3.1 (Kronecker's theorem). Suppose that $f \in L^{\infty}$. Then $H_{f}$ has finite rank if and only if there exists a nonzero analytic polynomial $p(z)$ such that $p f \in H^{\infty}$.

As is well known, for $f, g \in L^{\infty}$, Brown and Halmos [2] have shown that the product of two Toeplitz operators $T_{f}$ and $T_{g}$ is also a Toeplitz operator if and only if $\bar{f} \in H^{\infty}$ or $g \in H^{\infty}$. Axler et al. [1] have shown that the product $T_{f} T_{g}$ is a finiterank perturbation of a Toeplitz operator if and only if one of the operators $H_{\bar{f}}$ or $H_{g}$ has finite rank, where $f, g$ both are in $L^{\infty}$. We need the following lemma which may have been known before.

LEMMA 3.2. Let $A$ be a bounded linear operator on $H^{2}$. Suppose that $p(z)$ and $q(z)$ are nonzero analytic polynomials. If $T_{\bar{P}} A T_{q}$ has finite rank, then $A$ has finite rank. 
Proof. Factorize $q(z)$ as the product $q(z)=B(z) F(z)$ of a finite Blaschke product $B(z)$ and an outer function $F(z)$. Let $M=T_{\bar{p}} A T_{q} H^{2}$. Since $T_{p} A T_{q}$ has finite rank, $M$ is a finite dimension subspace of $H^{2}$. Since $F(z)$ is an outer function, closure $\left\{T_{F} H^{2}\right\}=H^{2}$. Thus

$$
\begin{aligned}
\operatorname{closure}\left\{T_{\bar{p}} A T_{q} H^{2}\right\} & =\operatorname{closure}\left\{T_{\bar{p}} A T_{B} T_{F} H^{2}\right\} \\
& =\operatorname{closure}\left\{T_{\bar{p}} A T_{B} H^{2}\right\}=M .
\end{aligned}
$$

This gives that $T_{\bar{p}} A T_{B}$ has finite rank and then

$$
T_{\bar{p}} A=T_{\bar{p}} A T_{\bar{B}} T_{B}=T_{\bar{p}} A T_{B} T_{\bar{B}} \bmod (F) .
$$

So $T_{\bar{p}} A$ has finite rank. By the same argument, we have that $A^{*}$ has finite rank. Hence $A$ has finite rank also. This completes the proof.

For $f \in L^{2}$, the Toeplitz operator $T_{f}$ and the Hankel operator $H_{f}$ are densely defined on $H^{2}$. For $f, g \in L^{2}$, Zheng [10] has given some conditions for the boundedness of the product of two Hankel operators. In the following theorem we will assume that the symbols of the Toeplitz operators lie in $\bigcap_{1<q<\infty} L^{q}$, and hence we extend Axler, Chang and Sarason's theorem.

THEOREM 3.3. For $f, g$, $h$ in $\bigcap_{1<q<\infty} L^{q}$ where $T_{f} T_{g}-T_{h}$ is a bounded operator on $H^{2}$, then

$$
T_{f} T_{g}=T_{h} \bmod (F)
$$

if and only if $h=f g$ and there is a nonzero analytic polynomial $A(z)$ such that

$$
A \bar{f} \in H^{2} \quad \text { or } \quad A g \in H^{2} .
$$

PROOF. First we prove the necessary part.

Let $k_{z}$ be the normalized reproducing kernel of $H^{2}$ at the point $z \in D$. We know that $k_{z}$ weakly converges to zero in $H^{2}$ as $z$ tends to the boundary of $D$. For $\xi \in \partial D$, $0<r<1$, by the hypothesis that $T_{f} T_{g}=T_{h} \bmod (F)$,

$$
\lim _{r \rightarrow 1}\left\langle T_{f} T_{g} k_{r \xi}, k_{r \xi}\right\rangle=\lim _{r \rightarrow 1}\left\langle T_{h} k_{r \xi}, k_{r \xi}\right\rangle
$$

It follows that $h=f g$ on $\partial D$. By Theorem 2.1, there is a nonzero analytic polynomial $A(z)$ such that $A \bar{f} \in H^{2}$ or $A g \in H^{2}$.

Next we prove the sufficient part.

If $h=f g$, there is a nonzero analytic polynomial $A(z)$ such that $A \bar{f} \in H^{2}$ or $A g \in H^{2}$.

Assume that $A g \in H^{2}$, then

$$
\left(T_{f} T_{g}-T_{h}\right) T_{A}=T_{f} T_{A g}-T_{A h}=T_{f g A-h A}=0 .
$$

Thus $T_{f} T_{g}=T_{h} \bmod (F)$ by Lemma 3.2. 
Assume that $A \bar{f} \in H^{2}$, then

$$
T_{\bar{A}}\left(T_{f} T_{g}-T_{h}\right)=T_{\bar{A} f} T_{g}-T_{\bar{A} h}=T_{\bar{A}(f g-h)}=0 .
$$

Hence $T_{f} T_{g}=T_{h} \bmod (F)$ by Lemma 3.2.

This completes the proof of the theorem.

TheOrEm 3.4. For $f_{1}, f_{2}, g_{1}, g_{2}$ in $L^{\infty}$ with $f_{1} g_{1}=f_{2} g_{2}$, then

$$
T_{f_{1}} T_{g_{1}}=T_{f_{2}} T_{g_{2}} \bmod (F),
$$

if and only if one of the following conditions holds:

(1) $H_{f_{1}}^{*} H_{g_{1}}$ and $H_{f_{2}}^{*} H_{g_{2}}$ are both finite-rank operators;

(2) there exist nonzero analytic polynomials $A_{1}(z), A_{2}(z), B_{1}(z)$ and $B_{2}(z)$ with $A_{1} \bar{B}_{1}=A_{2} \bar{B}_{2}$ such that

$$
A_{1} \bar{f}_{1}+A_{2} \bar{f}_{2} \in H^{\infty} \quad \text { and } \quad B_{1} g_{1}+B_{2} g_{2} \in H^{\infty} .
$$

PROOF. First we prove the 'only if' part. As

$$
T_{f_{1}} T_{g_{1}}-T_{f_{2}} T_{g_{2}}=H_{\bar{f}_{2}}^{*} H_{g_{2}}-H_{\bar{f}_{1}}^{*} H_{g_{1}},
$$

hence

$$
T_{f_{1}} T_{g_{1}}=T_{f_{2}} T_{g_{2}} \bmod (F)
$$

Equivalently,

$$
H_{\bar{f}_{2}}^{*} H_{g_{2}}-H_{f_{1}}^{*} H_{g_{1}}=0 \bmod (F) .
$$

Either $H_{f_{2}}^{*} H_{g_{2}}$ and $H_{f_{1}}^{*} H_{g_{1}}$ are both finite-rank operators or none of $H_{f_{2}}^{*} H_{g_{2}}$ and $H_{\bar{f}_{1}}^{*} H_{g_{1}}$ are finite-rank operators. If none of $H_{\bar{f}_{2}}^{*} H_{g_{2}}$ and $H_{\bar{f}_{1}}^{*} H_{g_{1}}$ are finite-rank operators, by Theorem 2.1, there exist nonzero analytic polynomials $A_{1}, A_{2}, B_{1}$ and $B_{2}$ such that

$$
A_{1} \bar{f}_{1}+A_{2} \bar{f}_{2}=h_{1} \in H^{\infty}
$$

or

$$
B_{1} g_{1}+B_{2} g_{2}=h_{2} \in H^{\infty} .
$$

Assume that $A_{1} \bar{f}_{1}+A_{2} \bar{f}_{2}=h_{1} \in H^{\infty}$, then $\overline{A_{1}} f_{1}+\overline{A_{2}} f_{2}=\overline{h_{1}}$.

Since $T_{f_{1}} T_{g_{1}}=T_{f_{2}} T_{g_{2}} \bmod (F)$,

$$
T_{\overline{A_{1}} f_{1}} T_{g_{1}}=T_{\overline{A_{1}} f_{2}} T_{g_{2}} \bmod (F) .
$$

This implies that

$$
T_{f_{2}} T_{\left(\bar{A}_{1} g_{2}+\bar{A}_{2} g_{1}\right)}=T_{\bar{h}_{1} g_{1}} \bmod (F)
$$


By Theorem 3.3, there is nonzero analytic polynomial $p(z)$ such that $p \overline{f_{2}} \in H^{\infty}$ or $p\left(\bar{A}_{1} g_{2}+\bar{A}_{2} g_{1}\right) \in H^{\infty}$. But $H_{\overline{f_{2}}}$ is not a finite-rank operator by the hypothesis, so $p\left(\bar{A}_{1} g_{2}+\bar{A}_{2} g_{1}\right) \in H^{\infty}$. Let $l=\max \left\{\operatorname{deg} \overline{A_{1}}, \operatorname{deg} \overline{A_{2}}\right\}, B_{1}=z^{l} p(z) \overline{A_{2}}, \quad B_{2}=$ $z^{l} p(z) \overline{A_{1}}$, so $B_{1}$ and $B_{2}$ are analytic polynomials such that

$$
B_{1} g_{1}+B_{2} g_{2} \in H^{\infty}
$$

and

$$
A_{1} \overline{B_{1}}=A_{2} \overline{B_{2}}
$$

If $B_{1} g_{1}+B_{2} g_{2}=h_{2} \in H^{\infty}$, by a similar argument, we obtain the same result.

Now we prove the 'if' part. Obviously, condition (1) is sufficient. Assume that there exist nonzero analytic polynomials $A_{1}, A_{2}, B_{1}$ and $B_{2}$ with $A_{1} \overline{B_{1}}=A_{2} \overline{B_{2}}$ such that $A_{1} \overline{f_{1}}+A_{2} \overline{f_{2}}=h_{1} \in H^{\infty}$ and $B_{1} g_{1}+B_{2} g_{2}=h_{2} \in H^{\infty}$.

Therefore,

$$
\begin{aligned}
T_{\bar{A}_{1}}\left(T_{f_{1}} T_{g_{1}}-T_{f_{2}} T_{g_{2}}\right) T_{B_{1}} & =T_{\bar{A}_{1} f_{1}} T_{g_{1} B_{1}}-T_{\bar{A}_{1} f_{2}} T_{g_{2} B_{1}} \\
& =\left(T_{\bar{h}_{1}}-T_{\bar{A}_{2} f_{2}}\right) T_{g_{1} B_{1}}-T_{A_{1} f_{2}} T_{g_{2} B_{1}} \\
& =T_{\bar{h}_{1} g_{1} B_{1}}-T_{\bar{A}_{2} f_{2}}\left(T_{h_{2}}-T_{B_{2} g_{2}}\right)-T_{\bar{A}_{1} f_{2}} T_{g_{2} B_{1}} \\
& =T_{\left(\bar{h}_{1} g_{1} B_{1}-\bar{A}_{2} h_{2} f_{2}\right)}+T_{\bar{A}_{2} f_{2}} T_{B_{2} g_{2}}-T_{\bar{A}_{1} f_{2}} T_{g_{2} B_{1}} .
\end{aligned}
$$

Note that, for any analytic polynomial $p(z)$, any $f \in L^{\infty}, T_{p} T_{f}=T_{f} T_{p} \bmod (F)$. Hence $T_{\bar{A}_{2} f_{2}} T_{B_{2} g_{2}}-T_{\bar{A}_{1} f_{2}} T_{g_{2} B_{1}}=0 \bmod (F)$. It is easy to calculate that $\bar{h}_{1} g_{1} B_{1}-$ $\bar{A}_{2} h_{2} f_{2}=0$. Thus,

$$
T_{\bar{A}_{1}}\left(T_{f_{1}} T_{g_{1}}-T_{f_{2}} T_{g_{2}}\right) T_{B_{1}}=0 \quad \bmod (F) .
$$

By Lemma 3.2, $T_{f_{1}} T_{g_{1}}=T_{f_{2}} T_{g_{2}} \bmod (F)$. This completes the proof.

COROLlary 3.5. For $f$ and $g$ in $L^{\infty}$, then

$$
T_{f} T_{g}=T_{g} T_{f} \quad \bmod (F),
$$

if and only if one of the following conditions holds:

(1) $H_{\bar{f}}^{*} H_{g}$ and $H_{\bar{g}}^{*} H_{f}$ are both finite-rank operators;

(2) there exist nonzero analytic polynomials $A_{1}(z), A_{2}(z), B_{1}(z)$ and $B_{2}(z)$ with $A_{1} \bar{B}_{1}=A_{2} \bar{B}_{2}$ such that

$$
A_{1} \bar{f}+A_{2} \bar{g} \in H^{\infty} \quad \text { and } \quad B_{1} g+B_{2} f \in H^{\infty} .
$$

THEOREM 3.6. For $f$ in $L^{\infty}$, the self-commutator

$$
T_{f}^{*} T_{f}-T_{f} T_{f}^{*}
$$

has finite rank if and only if one of the following conditions holds: 
(1) there is a nonzero analytic polynomial $p(z)$ such that $p f \in H^{\infty}$ and $p \bar{f} \in H^{\infty}$;

(2) there exist nonzero analytic polynomials $A$ and $B$ with $|A|^{2}=|B|^{2}$ such that

$$
A f+B \bar{f} \in H^{\infty} .
$$

PROOF. First we prove the 'only if' part. Suppose that

$$
T_{f}^{*} T_{f}-T_{f} T_{f}^{*}=H_{\bar{f}}^{*} H_{\bar{f}}-H_{f}^{*} H_{f}=0 \bmod (F) .
$$

Therefore, $H_{f}$ has finite rank if and only if $H_{\bar{f}}$ has finite rank.

If $H_{f}$ and $H_{\bar{f}}$ have finite rank, by Kronecker's theorem, there exist nonzero polynomials $p_{1}$ and $p_{2}$ such that $p_{1} \bar{f} \in H^{\infty}$ and $p_{2} f \in H^{\infty}$. Let $p(z)=p_{1} p_{2}$, so $p f$ and $p \bar{f}$ are both in $H^{\infty}$.

If none of $H_{\bar{f}}$ and $H_{f}$ have finite rank, by Theorem 3.4, there exist nonzero analytic polynomials $A$ and $B$ such that

$$
A f+B \bar{f}=h \in H^{\infty} .
$$

Therefore,

$$
\begin{aligned}
& T_{\bar{A}}\left(T_{\bar{f}} T_{f}-T_{f} T_{\bar{f}}\right) T_{B}=T_{\overline{A f}} T_{f B}-T_{\bar{A} f} T_{\bar{f} B} \\
& =\left(T_{\bar{h}}-T_{\bar{B} f}\right) T_{f B}-T_{\bar{A} f}\left(T_{h}-T_{A f}\right) \\
& =T_{(\bar{h} f B-\bar{A} f h)}-T_{\bar{B} f} T_{f B}+T_{\bar{A} f} T_{f A}
\end{aligned}
$$

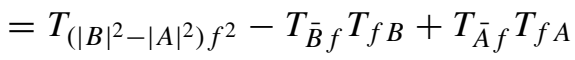

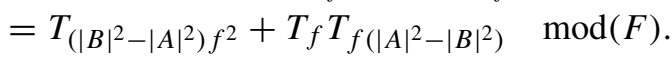

Since $T_{\bar{f}} T_{f}-T_{f} T_{\bar{f}}=0 \bmod (F), \quad T_{f} T_{f\left(|A|^{2}-|B|^{2}\right)}=T_{\left(|A|^{2}-|B|^{2}\right) f^{2} \bmod (F) . \quad \text { This }}$ implies that $|A|^{2}=|B|^{2}$ by Theorem 3.3 and the hypothesis follows. This completes the proof of the 'only if' part.

Next we prove the 'if' part. Obviously the condition 1 is sufficient. Suppose that condition (2) holds. That is, $|A|^{2}=|B|^{2}$ such that $A f+B \bar{f}=h \in H^{\infty}$, where $A$ and $B$ are nonzero analytic polynomials. Thus,

$$
\begin{aligned}
T_{\bar{A}}\left(T_{\bar{f}} T_{f}-T_{f} T_{\bar{f}}\right) T_{B} & =\left(T_{\bar{h}}-T_{\bar{B} f}\right) T_{f B}-T_{\bar{A} f}\left(T_{h}-T_{A f}\right) \\
& =T_{f(\bar{h} B-\bar{A} h)}+T_{f} T_{f\left(|A|^{2}-|B|^{2}\right)} \quad \bmod (F) \\
& =0 .
\end{aligned}
$$

Hence

$$
T_{\bar{f}} T_{f}-T_{f} T_{\bar{f}}=0 \quad \bmod (F),
$$

since Lemma 3.2. This completes the proof of the theorem.

THEOREM 3.7. For $f_{1}, f_{2}, f_{3}$ and $h$ in $L^{\infty}$, then

$$
T_{f_{1}} T_{f_{2}} T_{f_{3}}=T_{h} \bmod (F)
$$

if and only if one of the following conditions holds: 
(1) $H_{f_{1} f_{2}^{+}}^{*} H_{f_{3}}$ and $H_{f_{1}}^{*} H_{f_{2}^{-} f_{3}}$ are both finite-rank operators;

(2) there exist nonzero analytic polynomials $A_{1}, A_{2}, B_{1}$ and $B_{2}$ with $A_{1} \overline{B_{1}}+$ $A_{2} \overline{B_{2}}=0$ such that

$$
A_{1} \overline{f_{1} f_{2}^{+}}+A_{2} \overline{f_{1}}=r_{1} \in H^{2}
$$

and

$$
B_{1} f_{3}+B_{2} f_{2}^{-} f_{3}=r_{2} \in H^{2} .
$$

PROOF. We first prove the 'if' part. Since $h=f_{1} f_{2} f_{3}$,

$$
\begin{aligned}
T_{f_{1}} T_{f_{2}} T_{f_{3}}-T_{h} & =T_{f_{1} f_{2}^{+}}-T_{f_{1} f_{2}^{+} f_{3}}+T_{f_{1}} T_{f_{2}^{-} f_{3}}-T_{f_{1} f_{2}^{-} f_{3}} \\
& =-\left(H_{f_{1} f_{2}^{+}}^{*} H_{f_{3}}+H_{f_{1}}^{*} H_{\bar{f}_{2} f_{3}}\right) .
\end{aligned}
$$

Thus condition (1) implies that

$$
T_{f_{1}} T_{f_{2}} T_{f_{3}}=T_{h} \bmod (F) .
$$

Next we suppose that condition (2) is true. We have

$$
\begin{aligned}
T_{\overline{A_{1}}}\left(T_{f_{1}} T_{f_{2}} T_{f_{3}}-T_{h}\right) T_{B_{2}} & =T_{\overline{A_{1}} f_{1} f_{2}^{+}} T_{f_{3} B_{2}}+T_{\overline{A_{1}} f_{1}} T_{f_{2}^{-} f_{3} B_{2}}-T_{\overline{A_{1}} h B_{2}} \\
& =\left(T_{\overline{T_{1}}}-T_{\overline{A_{2}} f_{1}}\right) T_{f_{3} B_{2}}+T_{\overline{A_{1}} f_{1}}\left(T_{r_{2}}-T_{B_{1} f_{3}}\right)-T_{\overline{A_{1}}} h B_{2} \\
& =T_{\overline{r_{1}} B_{2} f_{3}+r_{2} \bar{A}_{1} f_{1}-\bar{A}_{1} B_{2} h}-T_{f_{1} f_{2}\left(\bar{A}_{2} B_{2}+\bar{A}_{1} B_{1}\right)} \quad \bmod (F) \\
& =0 .
\end{aligned}
$$

Hence

$$
T_{f_{1}} T_{f_{2}} T_{f_{3}}=T_{h} \bmod (F),
$$

from Lemma 3.2.

Next we prove the 'only if' part. If $T_{f_{1}} T_{f_{2}} T_{f_{3}}=T_{h} \bmod (F)$, by [3, Douglas theorem], $h=f_{1} f_{2} f_{3}$. Hence

$$
\begin{aligned}
T_{f_{1}} T_{f_{2}} T_{f_{3}}-T_{h} & =T_{f_{1} f_{2}^{*}} T_{f_{3}}+T_{f_{1}} T_{f_{2}^{-} f_{3}}-T_{f_{1} f_{2} f_{3}} \\
& =-\left(H_{f_{1} f_{2}^{+}}^{*} H_{f_{3}}+H_{\bar{f}_{1}}^{*} H_{\bar{f}_{2} f_{3}}\right) .
\end{aligned}
$$

If none of $H_{f_{1} f_{2}^{+}}^{*} H_{f_{3}}$ and $H_{f_{1}}^{*} H_{f_{2}^{-} f_{3}}$ have finite rank, by Theorem 2.1, there exist nonzero analytic polynomials $A_{1}, A_{2}, B_{1}$ and $B_{2}$ such that

$$
A_{1} \overline{f_{1} f_{2}^{+}}+A_{2} \overline{f_{1}}=r_{1} \in H^{2}
$$

or

$$
B_{1} f_{3}+B_{2} f_{2}^{-} f_{3}=r_{2} \in H^{2}
$$


Assume that

$$
A_{1} \overline{f_{1} f_{2}^{+}}+A_{2} \overline{f_{1}}=r_{1} \in H^{2}
$$

then

$$
\begin{aligned}
T_{\bar{A}_{1}}\left(T_{f_{1}} T_{f_{2}} T_{f_{3}}-T_{h}\right) & =T_{\bar{A}_{1}}\left(T_{f_{1} f_{2}^{+}} T_{f_{3}}+T_{f_{1}} T_{f_{2}^{-} f_{3}}-T_{\bar{A}_{1} h}\right) \\
& =\left(T_{\bar{r}_{1}}-T_{\bar{A}_{2} f_{1}}\right) T_{f_{3}}+T_{\bar{A}_{1} f_{1}} T_{f_{2}^{-} f_{3}}-T_{\bar{A}_{1} h} \\
& =T_{f_{1}} T_{\left(\bar{A}_{1} f_{2}^{-} f_{3}-\bar{A}_{2} f_{3}\right)}+T_{\bar{r}_{1} f_{3}-\bar{A}_{1} h} \quad \bmod (F) .
\end{aligned}
$$

This implies that

$$
T_{f_{1}} T_{\left(\bar{A}_{1} f_{2}^{-} f_{3}-\bar{A}_{2} f_{3}\right)}=T_{\bar{A}_{1} h-\bar{r}_{1} f_{3}} \bmod (F) .
$$

By Theorem 3.3 and the hypothesis, there is a nonzero analytic polynomial $p$ such that

$$
p\left(\bar{A}_{1} f_{2}^{-} f_{3}-\bar{A}_{2} f_{3}\right) \in H^{2} .
$$

Let $l=\max \left\{\operatorname{deg} \bar{A}_{1}, \operatorname{deg} \bar{A}_{2}\right\}, B_{1}=-z^{l} p(z) \bar{A}_{2}(z)$ and $B_{2}=z^{l} p(z) \bar{A}_{1}(z)$. Then $B_{1}$ and $B_{2}$ are both nonzero analytic polynomials with

$$
A_{1} \overline{B_{1}}+A_{2} \overline{B_{2}}=0
$$

such that

$$
B_{1} f_{3}+B_{2} f_{2}^{-} f_{3} \in H^{2}
$$

This complete the proof.

\section{Acknowledgement}

The author thanks D. Zheng for his warm hospitality during his visiting Vanderbilt University.

\section{References}

[1] S. Axler, S. Y. A. Chang and D. Sarason, 'Product of Toeplitz operators', Integral Equations Operator Theory 1 (1978), 283-309.

[2] A. Brown and P. R. Halmos, 'Algebraic properties of Toeplitz operators', J. Reine Angew. Math. 213 (1963), 89-102.

[3] R. G. Douglas, Banach Algebra Techniques in Operator Theory (Academic Press, New York, London, 1972).

[4] - Banach Algebra Techniques in the Theory of Toeplitz Operators, Regional Conference Series in Mathematics, 15 (American Mathematical Society, Providence, RI, 1972).

[5] C. Gu, 'Products of several Toeplitz operators', J. Funct. Anal. 171 (2000), 483-527.

[6] _ ' 'On operators commutiong with Toeplitz operators modulo the finite rank operators', J. Funct. Anal. 215 (2004), 178-205. 
[7] K. Guo and D. Zheng, 'The distribution function inequality for a finite sum of finite products of Toeplitz operators', J. Funct. Anal. 218 (2005), 1-53.

[8] N. K. Nikolskii, Treatise on the Shift Operator (Nauka, Moscow, 1980); English transl. Grundlehren Math. Wiss., Vol. 273 (Springer-Verlag, Berlin, 1986).

[9] V. Peller, Hankel Operators and Their Applications, Springer Monographs in Mathematics (Springer, New York, 2003).

[10] D. Zheng, 'The distribution function inequality and products of Toeplitz operators and Hankel operators', J. Funct. Anal. 138 (1996), 477-501.

XUANHAO DING, College of Science, Chong Qing Technology and Business University, Chong Qing, 400067, PR China

e-mail: dxuanhao@gmail.com 